\title{
How does a reaction path branching take place? A classification of bifurcation events
}

\author{
Wolfgang Quapp ${ }^{*, 1}$ \\ Mathematisches Institut, Universität Leipzig, Augustus-Platz, Leipzig D-04109, Germany \\ Received 17 September 2003; revised 14 October 2003; accepted 15 October 2003
}

\begin{abstract}
Valley-ridge-inflection points (VRI) of a potential energy surface (PES) may have a strong relation to bifurcations of chemical reaction pathways. We explain how a reaction path has to be defined to describe the bifurcation of a 'valley' of the PES. We propose a classification of different VRI events: valley bifurcation, ridge bifurcation, and the mixed case in between. Chemical implications of the different cases are discussed.

(C) 2004 Elsevier B.V. All rights reserved.
\end{abstract}

Keywords: Reaction path bifurcation; Projected gradient; Valley-ridge-inflection point; Pitchfork bifurcation; Classification of VRIs

\section{Introduction}

The vibrations of any molecule around its equilibrium structure may be determined by the harmonic oscillator approximation to generate the normal modes [1]. The PES of the electronic ground state for this model is an $n$ dimensional paraboloid where $n$ is the number of internal degrees of freedom [2]. Border-line cases studied are the large amplitude motion of light atoms, like an $\mathrm{H}$ atom in HCN bending $[3,4]$, or the case of quasilinear molecules, like HCNO [5-7]. At higher vibrational excitations, however, every molecule leaves the nearly harmonic basin of the PES. Stationary vibrational states change to moving wavepackets [8]. The landscape becomes a terra incognita. A useful picture of how a chemical reaction proceeds that is familiar to most chemists is one where a wavepacket transforms the reactant into the product by generally taking the 'lowest energy' pathway available passing through a transition state-the highest energy point on the pathway. Although obviously an oversimplification-given enough excitation wavepackets can wander all over the PES- the 'reaction path' (RP) concept is very appealing. Usually, this concept uses the RP in any curvilinear shape giving up the normal mode directions of the basins. The change of normal

\footnotetext{
* Tel.: +49-341-9732153; fax: +49-341-9732199.

E-mail address: quapp@rz.uni-leipzig.de (W. Quapp).

${ }^{1}$ http://www.mathematik.uni-leipzig.de/MI/quapp
}

modes along the RP is more often than not continuous if going along the curvilinear valley up to the saddle point (SP) of the PES [9]. However, there are other, disarranging possibilities in molecules: (i) the valley can end at the slope, thus, the transition state is not at the top of the reactant valley $[10,11]$, or (ii) there is a branching of the reaction channel usually caused by a symmetry break $[12,13]$. The latter point (ii) shall be treated in this article. It is then assumed that a bifurcation of a PES valley (or of a ridge) is connected with valley-ridge-inflection (VRI) points.

\section{Valley-ridge-inflection points}

It is helpful to consider that RP branching is in many cases connected with the emergence of a special class of points of the PES, the VRI points [14-19]; we first give a definition of these points.

A VRI point is that point in the configuration space where, orthogonally to the gradient, at least one main curvature of the PES becomes zero. This has two conditions

(i) one eigenvalue of the Hessian must be zero, and

(ii) the gradient is orthogonal to the corresponding zeroeigenvector.

VRI points in the narrow sense of this definition are given independently of any curve definition. By contrast, 
VRI points are generally not identical with bifurcation points (BP) of any curve. Only the RGF curves (to be defined below) bifurcate at VRI points! Usually, VRI points represent non-stationary points of the PES [16].

\section{Which reaction path can bifurcate at a VRI point?}

\subsection{Probing the steepest descent}

Goodness is to human nature like flowing downward to water. Meng-tse (c.300 BCE) (for Brenda and Manfred)

Looking for the potential of the usual RP following to locate bifurcations we have to treat firstly the IRC [20], see also Refs. [21-23]. It is a simple RP concept by the union of the steepest descents from SP down to reactant and product basins, respectively. With arc length $s$ for the curve parameter, a steepest descent curve $\mathbf{x}(s)$ is defined by

$$
\frac{\mathrm{d} \mathbf{x}(s)}{\mathrm{d} s}=-\frac{\mathbf{g}(\mathbf{x}(s))}{\|\mathbf{g}(\mathbf{x}(s))\|} .
$$

However, because of its simplicity of using the gradient $\mathbf{g}$ only, the IRC does not detect how the level lines change from convex to concave behavior: by means of example, Fig. 1. The path through $\mathrm{SP}_{1}$ is a hanging valley. The IRC from $\mathrm{SP}_{1}$ leads straight downhill to $\mathrm{SP}_{2}$. It goes orthogonally to the levels, and this is the upshot of the state-of-the-art! The definition does not use the curvature of the levels, and it does not test any valley floor character of its pathway. But after a change of levels from convex to concave behavior

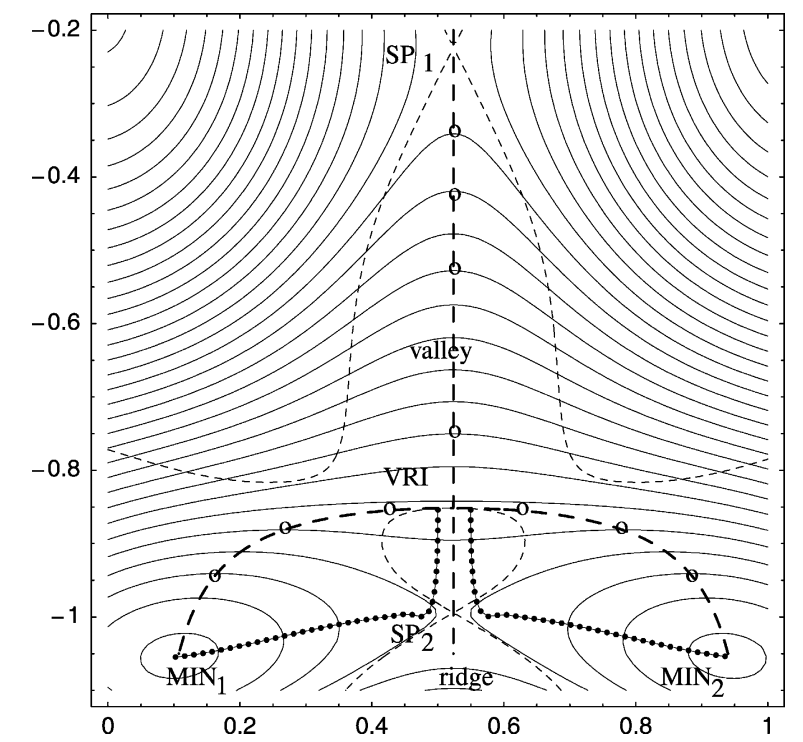

Fig. 1. Equipotential lines of a model potential energy surface. Thin dashes depict the border between valley and ridge. The valley-ridge-inflection point $(\mathrm{VRI})$ is at $(\pi / 6,-0.85)$. RGFs are bold dashes and steepest descent lines starting near the VRI are the connected fat points. A search for minima orthogonal to the gradient direction of the IRC (the $y$-axis at $\mathrm{SP}_{1}$ ) gives the points of the 'o' symbols. the IRC ceases to be a useful RP model. In the convex region it can serve as an RP model, however, actually not in the ridge region.

Included in Fig. 1 is the border between ridge and valley regions [13]. That border is defined by

$\mathbf{g}^{\mathrm{T}} \mathbf{A g}=0$

where $\mathbf{A}$ is the adjoint matrix [17] to the Hessian matrix $\mathbf{H}$. $\mathbf{A}$ is defined as $\left((-1)^{i+j} m_{i j}\right)^{\mathrm{T}}$ where $m_{i j}$ is the minor of $\mathbf{H}$ obtained by deletion of the $i$ th row and the $j$ th column from $\mathbf{H}$, and taking the determinant. The superscript $\mathrm{T}$ denotes the transposition.

Any small perturbation of the IRC calculation also does not push immediately the steepest descent into a side valley, but it goes further through the ridge region in most cases. In Fig. 1 two example pathways are included. The VRI point is at $(0.52,-0.85)$. The steepest descents which start there close to the symmetric IRC will diverge from the IRC very slowly $[24,25]$. The distorted pathways run past the lower $\mathrm{SP}_{2}$ only, to directly find a minimum. Pay attention to the often assumed opinion that the IRC splits at the VRI point. For example, this is explicitly said in Ref. [26], or drawn in papers of Sordo et al. [27]; but it is not true.

From a mathematical point of view, the IRC pathway Eq. (1) is defined by an autonomous system of differential equations for a tangent vector along the curve searched for. Its solution is unique. Therefore, no bifurcation can occur before reaching the next stationary point [28]. Hence, no branching of PES valleys will be truly described by following the IRC. A further lack of the IRC concept is that the IRC usually miss asymmetric VRIs at all [13]; however, see an exceptional result in Ref. [14].

\subsection{Probing the distinguished coordinate (or RGF)}

Some 20 years ago an alternate concept to the IRC was proposed: choose a driving coordinate along the 'reaction valley' of the minimum, go a step in this direction, and perform an energy optimization of the residual coordinates [29,30]. A combination of the distinguished coordinate method starting at the SP and steepest descent was also used [31]. In Fig. 1 one can study the effect of the method: we depict some minima found by small circles. Starting at the $\mathrm{SP}_{1}$, one indeed obtains the valley downhill, and after the VRI point the method is able to find the parting of the ways of the valley bifurcation. The IRC continues to go further downhill the ridge, but a minimization orthogonal to the $y$ direction splits into two subdimensional minima at every $y$ level after VRI. It finds the two valley branches to the left and to the right. Thus, we have found a model of the RP bifurcation: fortunately, the VRI point is the BP of the searched curve. Note that the two branches do not leave the former IRC under an acute [12] or asymptotic small [32] angle (as many theoreticians still assume!). The branches 
usually split in an orthogonal kind. At the beginning, the outgoing tines are collinear.

Warning: the two branches found by the distinguished coordinate method after VRI are a fine RP bifurcation model. However, we do not know exactly the 'direction' of the bifurcating valleys. Because, we use the orthogonal direction to the $y$-axis for the search being orthogonal to the former IRC direction. The distinguished coordinate paths only follow the outgoing valleys qualitatively. The pathways are usually not valley floors (whatever this may be). They follow a convex valley-like situation of the level lines down to the minima. Thus, they do what we want.

The methodology of the distinguished coordinate method was truly criticized by some workers, see for example by Müller and Brown [33], Williams and Maggiora [34], and Cioslowski et al. [35]. They found samples, where the distinguished coordinate method fails: it cannot follow the path over a turning point (TP) where the ascent of the curve changes to descent, or vice versa. More recently, the method was transformed into a new mathematical form [36]. The former limitations do not stem from failures of the approximation of the defined curve but are manifestations of the concept of minimization, orthogonal to the distinguished direction. If also the 'switch' to a maximization is allowed, then the TP problem disappears. It was realized by the method to follow the reduced gradient (RGF) [17,36-38]. RGF finds a curve where the selected gradient direction comes out equally at every curve point, $\mathbf{x}(t)$

$\mathbf{g}(\mathbf{x}(t)) /\|\mathbf{g}(\mathbf{x}(t))\|=\mathbf{r}$,

where $\mathbf{r}$ is the unit vector of a search direction, and $\mathbf{g}$ is the gradient of the PES. RGF is a favorable approach for RP branching, because the VRI points of the PES are the BPs of special RGF curves. In Figs. 1-3 we use RGFs to mark the VRI points with valley- or ridge bifurcations. Equivalent curves to RGF are also obtained by the global Newton method (Branin curves [39], see also Ref. [17]). Branin's method is additionally well adapted to exactly calculate symmetric VRI points [17]. There is a successful application to find VRIs of $\mathrm{H}_{2} \mathrm{O}$ [18], $\mathrm{H}_{2} \mathrm{~S}, \mathrm{H}_{2} \mathrm{Se}$, and $\mathrm{H}_{2} \mathrm{CO}$ [19], and $\mathrm{C}_{2} \mathrm{H}_{5}^{+}[40]$.

\section{Classification of VRI regions}

\subsection{Valley bifurcation}

Fig. 1 shows the valley bifurcation downhill starting at SP: the usual case of which chemists recently are interested [41]. The two branches lead to two product minima. At the VRI point the chemical system comes to its crossroads. The VRI point is on a valley-pitchfork (vpVRI) bifurcation.

Analogously, the uphill case of a bifurcating valley is often studied, see Refs. [12,19] for the case of $\mathrm{H}_{2} \mathrm{CO}$, and Ref. [13] for a legion of further references. In the uphill

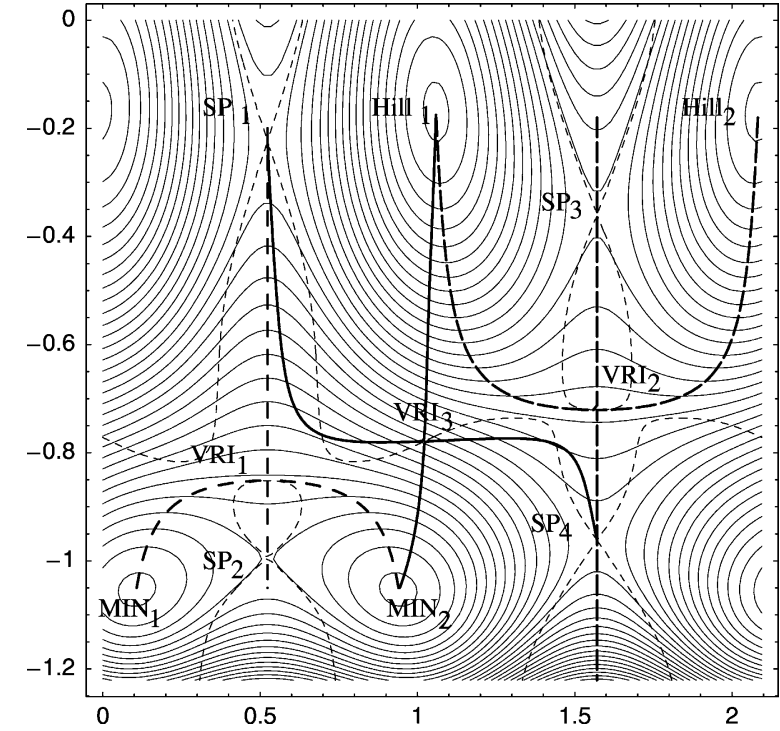

Fig. 2. 2D model PES with three different VRI regions. RGFs between interesting stationary points are bold dashes (two tri-tined pitchforks on the left and the right hand side) and the bold full branches in the center meeting at $\mathrm{VRI}_{3}$. Thin dashed lines are the border between valleys and ridges of the PES. Note that the axes are not equally scaled.

case, the two outer tines of the pitchfork being valleys lead to two SPs with a higher SP of index two in between [42]. For a figure of the uphill case cf. the remark at the end of Section 4.2.

\subsection{Ridge bifurcation}

Ridges are interesting in theoretical chemistry by itself because they are the prerequisites for some reaction rate theories using the 'dividing surface' of the transition state

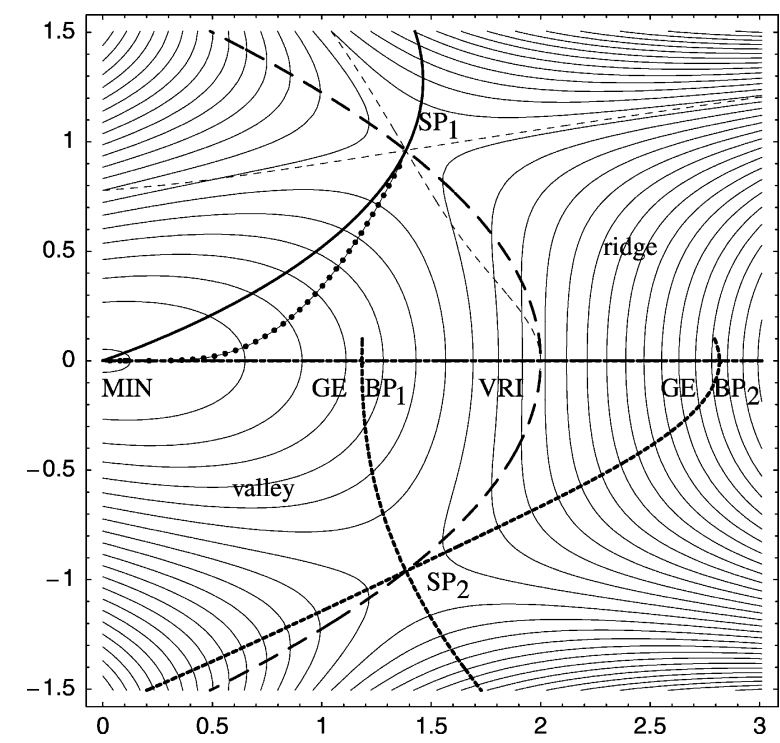

Fig. 3. 2D model PES with VRI region of a descending, bifurcating ridge. An RGF depicting this is shown by bold dashes. Another RGF between $\mathrm{MIN}$ and $\mathrm{SP}_{1}$ is the bold line, and the IRC from $\mathrm{SP}_{1}$ is the line with dots. Thin dashed lines show above the border between valley and ridge of the PES. Below there are Gradient Extremals (GE) included by dense bullets. 
[43]. There is a generalized concept of the transition state in form of a special potential ridge line [44].

If we start in Fig. 2 at $\mathrm{SP}_{4}$ in $y$ direction uphill and do not minimize, but maximize, we obtain the ridge of the PES. At point $(1.57,-0.73)$, the $\mathrm{VRI}_{2}$, the uphill case of a bifurcating ridge is observed. The two outer tines being ridge branches lead to two SPs of index two, or hills. The VRI point is on a ridge-pitchfork (rpVRI) bifurcation. From $\mathrm{SP}_{3}$ down, between the two hills, there is of course a colvalley. What happens with the valley at the VRI point, $\mathrm{VRI}_{2}$ ? It is a situation neither discussed in chemistry nor in spectroscopy up to date. The valley disappears totally. It is quite more disturbing than the situation given by an ending valley at the slope of a still convex region $[10,11,13]$. Minimization orthogonal to $y$ direction at a point below the VRI point cannot find a minimum anywhere in the neighbourhood. If at all, the minimization may jump to the valley-bifurcation at the left hand side of Fig. 2. Consequently, a mode orthogonal to $y$-axis which may vibrate orthogonally at transition state, $\mathrm{SP}_{3}$, will be destabilized and destroyed at VRI. The definition of an RP by the distinguished coordinate breaks down, in this case, as well as that of the IRC.

Fig. 3 shows a test potential with a bifurcating ridge leading downhill. The two bifurcating branches connect the 'main' ridge on $x$ axis with the SPs. The minimum valley leads uphill along the $x$-axis and ends at the VRI point, again totally. It does not bifurcate? At least neither for RGF lines, nor for the IRC. From MIN to SP there can be drawn a family of RGFs which make the connection, but all of them start at MIN, and all have a different acute angle to $x$-axis. (One member of this family is shown.) Also the two IRCs from the two SPs meet at MIN under an asymptotic, tangential direction to $x$-axis. To define a valley bifurcation in this case, we can use the more complicated tool of gradient extremals $[13,45]$. $\mathrm{BP}_{1}$ of a $\mathrm{GE}$ is the point where the MIN valley splits into three valleys: the two outer valleys to the SPs, and the central cirque [46] going further uphill to VRI. Analogously, the $\mathrm{BP}_{2}$ of a GE is the point where the ridge from the right hand side splits into three ridges: the two outer ridges to the SPs, and the central cliff [46] going further downhill to VRI. What is the true RP model--IRC, RGF, GE, or any other definition? This may depend upon the aim of the treatment. Note that the GE does not bifurcate at the VRI point, in general. It is well demonstrated on the given surface.

Chemical examples fulfilling the model PES of Fig. 3 are the transition state region of the $\mathrm{F}+\mathrm{H}_{2} \rightarrow \mathrm{FH}+\mathrm{H}$ rearrangement collision [47], as well as the analogous reaction $\mathrm{Cl}+\mathrm{H}_{2} \rightarrow \mathrm{ClH}+\mathrm{H} \quad$ [48], and possibly the dissociation reaction of the methaniminium cation, $\mathrm{CH}_{2} \mathrm{NH}_{2}^{+} \rightarrow \mathrm{CHNH}^{+}+\mathrm{H}_{2}$ [49], see Fig. 2(b) there.

Remark: a multiplication of the test surface by minus one will change it into a sample for the case of an uphill bifurcating valley for Section 4.1.

\subsection{Border-line case: 'flat' branches}

If there are the two possibilities of a pitchfork of both, valleys (say uphill) as well as ridges (say downhill), then there should be the border-line case of two 'flat' outer tines of the pitchfork. The case is indeed met in Lasorne et al. [50] for a PES model of the isomerization of $\mathrm{H}_{3} \mathrm{CO} \rightarrow \mathrm{H}_{2} \mathrm{COH}$, see Fig. 3 there. The two at tines are neither valleys nor ridges, but simply a flat equipotential line. They form a degenerate version of the extremal solution of minima $=$ valleys, or maxima $=$ ridges: the extremum along a 'flat potential' is the full line.

A second example is the torsional and wagging vibration of methylamine, $\mathrm{H}_{3} \mathrm{C}-\mathrm{NH}_{2}$ [51], cf. also Ref. [45], Figs. 5-7 there. The wagging mode direction shows the border-line case of a flat pitchfork. The flat tines lead to two SPs. They are the border of an 'isomerization channel'. The ridge in between uphill leads to a hill.

\subsection{Mixed bifurcation}

We observe a further kind of bifurcation in the center of Fig. 2. The special RGF curve shown by bold lines connects minimum $\mathrm{MIN}_{2}$ with $\mathrm{Hill}_{1}$, an $\mathrm{SP}$ of index two, as well as $\mathrm{SP}_{1}$ with $\mathrm{SP}_{4}$. The search direction used is $\mathbf{r}=(0.238,0.975)$. The special $\mathrm{RGF}$ passes $\mathrm{VRI}_{3}$ at $(1.02,-0.78)$. Near the minimum it describes a cirque pathway [46], and it changes to a ridge path after VRI uphill. The RGF curve bifurcates at $\mathrm{VRI}_{3}$ but it is not the usual pitchfork picture. The character is mixed. The valley from minimum continues to the right hand side to be a valley (up to a TP where also the valley character ends here). So, the RP character of the minimum cirque path can be continued as a valley after VRI. The ridge line from maximum continues to the left hand side to be a ridge (down to a TP where also the ridge character ends here). We may classify the VRI point to be of a valley-ridge touching kind (mixVRI). The characteristic of the mixed bifurcation is that the borderline between valleys and ridges intersects the outgoing branches transversally, not tangentially, as it does in the pitchfork cases, cf. Fig. 2.

In a triatomic molecule, $\mathrm{HCN}$, there are observed such mixVRI points recently [13]. Usually, mixed VRIs are asymmetric VRIs [13].

\section{Conclusions}

The mathematical description of RP branching is of high theoretical interest. From the author's viewpoint, it is one of those questions which now requires closer consideration in PES computational chemistry cite

'The rate of a reaction can be estimated by transition state theory from the energy, structure, and vibrational frequencies of the transition state. Reaction path 
following can identify with some certainty the reactants and products connected by the transition state, unless the path branches. If the branching occurs before the transition state, there will be a separate transition state for each branch, and transition state theory can be used to estimate the relative rates. If the branching occurs after the transition state,..., the branching ratio cannot be determined by transition state theory, but depends on the nature of the potential energy surface as it descends from the transition state toward the different products...' [41].

The IRC or generally steepest descent are not adapted to serve as an RP model for RP bifurcation. If there is a continuous valley from SP to minimum (convex isopotential hypersurfaces with respect to the minimum) the IRC may best serve as an RP model. However, in case of the existence of concave VRI regions along the IRC progress, one has to look for other RP models or modifications which may bifurcate at the VRI point.

A promising model is the RGF approach using the VRI points of the PES. If at the VRI point a valley shows a pitchfork bifurcation, or if it is a mixed VRI point then the corresponding RGF branches may be suited to a well-behaved RP model. The usual (more or less orthogonal) pitchfork branching of RGFs orthogonal to the IRC is, admittedly, not a very illuminating description of the theoretical chemist's imagination of a 'tangential' branching. However, the nature of branching defies that conventional description. The nature of branching is a crossroad like structure. Fig. 4 shows the nature of a biological branching for comparison: here the 'ridges' of the cactus (the ribs) also do not bifurcate tangentially. Back to chemistry: despite its importance, a theory of RP bifurcations is still in its infancy. The two branches of RGF leading away and being valley lines may be the border of a 'reaction channel'. Other lines of the channel will be obtained by a slight change of the search parameter $\mathbf{r}$ of Eq. (3). Members of such a family

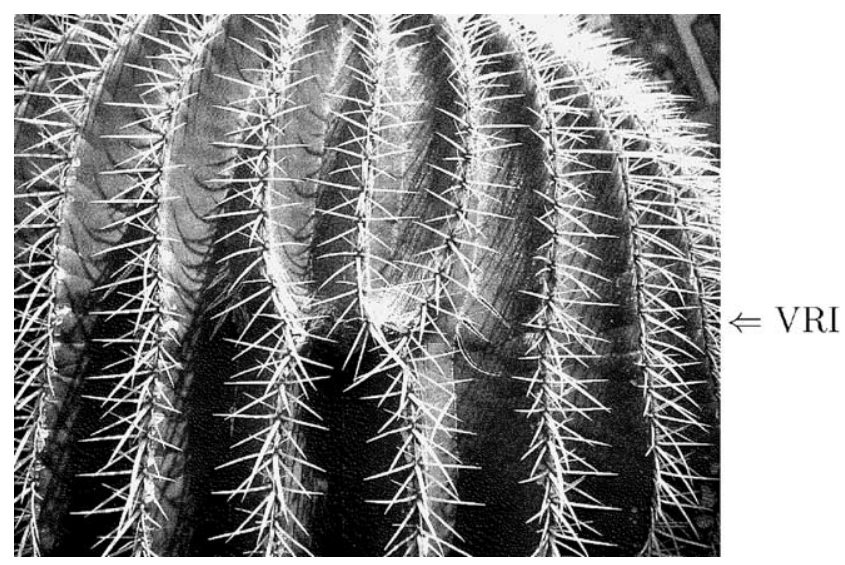

Fig. 4. Echinocactus grusonii with an 'uphill ridge bifurcation' on the surface (Botanischer Garten Leipzig, photo: Ute Jahn). are curves without the corner, in contrast to the RGF through the VRI point. Note that RGF curves were recently used to realize a calculation of an RP Hamiltonian [52].

If a turning point emerges, the RGF ceases to be a good RP model as well. Again, another search direction $\mathbf{r}$ may grasp a better line for an RP model [45]. If one finds a VRI with a ridge pitchfork then the discussed geometrical models of an RP cease to be meaningful at all. From a more general point of view, any line from a minimum to an SP of index one can be an RP model if two conditions are fulfilled: (i) the energy is monotonously increasing along the line, and (ii) the line always leads through valley regions. (A hypothetical straight line from $\mathrm{MIN}_{1}$ to $\mathrm{SP}_{1}$ in Fig. 1, however, is not such an RP model because it intersects a ridge. In Fig. 3 it is such an RP model.)

In more than two dimensions the VRI definition is valid as well. RGFs also bifurcate there, but there is generally a high dimensional manifold of VRI points [17-19,40]. However, the simple classification of valleys and ridges in $2 \mathrm{D}$ falls to pieces and opens to a wide field of intermediate cases [53].

\section{Outlook}

There was a speculation on the meaning of VRI for high overtones using the example $\mathrm{H}_{2} \mathrm{O}$ [18]. However, it seems that it is to reject by the experimental assigns of high local modes of water. So, the discussion of the meaning of VRI for spectroscopy is still open.

There is a recent remarkable experiment with a stimulated intramolecular vibrational energy redistribution (IVR) [42]. It leads to a chemical reaction path with an obvious bifurcation at the slope of the PES, the dissociation of diazomethane, $\mathrm{CH}_{2} \mathrm{~N}_{2}$. In our opinion, the result [42] may be a cornerstone for an experimental foundation of the theory of RP bifurcations, because usually, IVR is defined in terms of the local density of states, but not in terms of the local PES directly, cf. [54] and references therein.

\section{Acknowledgements}

This manuscript is submitted in honor of Brenda and Manfred Winnewisser. I am sincerely indebted for their friendship over the years, before and after the German unification. The work was supported by the Deutsche Forschungsgemeinschaft. Gratitude is expressed to M. Hirsch for stimulating discussions, and Prof. D. Heidrich for critically reading the manuscript, and for a long, fruitful cooperation. 


\section{Appendix}

The test surface used in Figs. 1 and 2 is

$$
\begin{aligned}
E(x, y)= & 44730.4129-66786.5363 \cos (y)+26352.6908 \\
& \times \cos (2 y)-3117.3613 \cos (4 y)+659.3217 \cos (6 y) \\
& +621.9640 \sin (3 x) \sin (y)-138.3050 \sin (3 x) \\
& \times \sin (2 y)-111.5488 \cos (8 y)+41.8227 \sin (3 x) \\
& \times \sin (4 y)-7.7979 \sin (3 x) \sin (6 y)+9.9258 \cos (6 x) \\
& -19.0681 \cos (6 x) \cos (y)+600 \cos (6 x) \cos (2 y) \\
& -500 \sin (3 y)
\end{aligned}
$$

It is a modified version of a $2 \mathrm{D}$ model surface of the torsional and wagging vibration modes of methylamine, $\mathrm{H}_{3} \mathrm{C}-\mathrm{NH}_{2}$ [51], cf. also [45]. The two pitchforks are solutions of the RGF Eq. (3) with $\mathbf{r}=(0,1)$, thus, $E_{x}=0$. The RGF line of the mixed behavior is found by $E_{x}-$ $0.244 E_{y}=0$.

The test surface used in Fig. 3 is $E(x, y)=$ $\mathrm{x}^{2}+\left(6-3 x-y^{2}\right) y^{2}$.

The pitchfork is solution to $\mathbf{r}=(1,0)$, thus, $E_{y}=0$. The special RGF line (selected from a family of lines) leading to SP is $0.91 E_{x}-0.42 E_{y}=0$.

\section{References}

[1] E.B. Wilson, J.C. Decius, P.C. Cross, Molecular Vibrations, McGrawHill, New York, 1955.

[2] B.P. Winnewisser, J.K.G. Watson, J. Mol. Spectrosc. 205 (2001) 227-231.

[3] J.T. Hougen, P. Bunker, J.W.C. Johnes, J. Mol. Spectrosc. 34 (1970) 136-172.

[4] W. Quapp, S. Klee, G.Ch. Mellau, S. Albert, A. Maki, J. Mol. Spectrosc. 167 (1994) 375-382. W. Quapp, V. Melnikov, G.Ch. Mellau, J. Mol. Spectrosc. 211 (2002) 184-197.

[5] K. Yamada, M. Winnewisser, Z. Naturf. 31 a (1976) 136-144.

[6] B.P. Winnewisser, in: Molecular Spectroscopy: Modern Research, vol. III, Academic Press, Orlando, 1985, pp. 321-419, Chapter 6. W. Quapp, B.P. Winnewisser, J. Math. Chem., 14, 1993, pp. 259-285.

[7] W. Quapp, S. Albert, B.P. Winnewisser, M. Winnewisser, J. Mol. Spectrosc. 160 (1993) 540-553.

[8] A.H. Zewail, J. Phys. Chem. 104 (2000) 5660-5694.

[9] W. Miller, N.C. Handy, J.E. Adams, J. Chem. Phys. 72 (1980) 99-112.

[10] F. Jensen, Theor. Chem. Acc. 99 (1998) 295-300

[11] S. Shaik, H.B. Schlegel, S. Wolfe, Theoretical Aspects of Physical Organic Chemistry, The SN2 Mechanism, Wiley, NewYork, 1992, Fig. 2.12, p. 79

[12] J. Baker, P.M.W. Gill, J. Comput. Chem. 9 (1988) 465-475.

[13] W. Quapp, M. Hirsch, D. Heidrich, Theor. Chem. Acc. (2003) accepted.

[14] T. Taketsugu, D.J. Wales, Mol. Phys. 100 (2002) 2793-2806.

[15] H. Metiu, J. Ross, R. Silbey, T.F. George, J. Chem. Phys. 61 (1974) 3200-3209.
[16] P. Valtazanos, K. Ruedenberg, Theoret. Chim. Acta 69 (1986) 281-307.

[17] W. Quapp, M. Hirsch, D. Heidrich, Theoret. Chem. Acc. 100 (1998) 285-299.

[18] M. Hirsch, W. Quapp, D. Heidrich, Phys. Chem. Chem. Phys. 1 (1999) 5291-5299.

[19] W. Quapp, V. Melnikov, Phys. Chem. Chem. Phys. 3 (2001) 2735-2741.

[20] K. Fukui, J. Phys. Chem. 74 (1970) 4161-4163.

[21] A. Tachibana, K. Fukui, Theoret. Chim. Acta 49 (1978) 321-347.

[22] W. Quapp, D. Heidrich, Theoret. Chim. Acta 66 (1984) 245-260.

[23] B.L. Garrett, M.J. Redmon, R. Steckler, D.G. Truhlar, K.K. Baldridge, D. Bartol, M.V. Schmidt, M.S. Gordon, J. Phys. Chem. 92 (1988) 1476-1488

[24] H.B. Schlegel, J. Chem. Soc., Faraday Trans. 90 (1994) 1569-1574.

[25] T. Yanai, T. Taketsugu, H. Hirano, J. Chem. Phys. 107 (1997) 1137-1146. Y. Kumeda, T. Taketsugu, J. Chem. Phys. 113 (2000) 477-484.

[26] G.V. Shustov, A. Rauk, J. Org. Chem. 63 (1998) 5413-5422.

[27] P. Braña, B. Menéndez, T. Fernández, J.A. Sordo, J. Phys. Chem. 104 (2000) 10842-10854. H. Valdés, J.A. Sordo, Chem. Phys. Lett. 333 (2001) 169-180.

[28] W. Quapp, J. Chem. Soc., Faraday Trans. 90 (1994) 1607-1609.

[29] C.W. Bauschlicher, H.F. Schaefer III, C.F. Bender, J. Am. Chem. Soc. 98 (1976) 1653-1658.

[30] R. Czerminski, R. Elber, J. Chem. Phys. 92 (1990) 5580-5601.

[31] R. Steckler, D.G. Truhlar, J. Chem. Phys. 93 (1990) 6570-6577.

[32] P.Y. Ayala, H.B. Schlegel, J. Chem. Phys. 107 (1997) 375-384. scheme 7.

[33] K. Müller, Angew. Chem. 92 (1980) 1-14. K. Müller, L.D. Brown, Theor. Chim. Acta 53 (1979) 75-93.

[34] I.H. Williams, G.M. Maggiora, J. Mol. Struct. (Theochem) 89 (1982) $365-378$.

[35] J. Cioslowski, A.P. Scott, L. Radon, Mol. Phys. 91 (1997) 413-420.

[36] W. Quapp, M. Hirsch, O. Imig, D. Heidrich, J. Comput. Chem. 19 (1998) 1087-1100.

[37] W. Quapp, J. Comput. Chem. 22 (2001) 537-540.

[38] M. Hirsch, W. Quapp, J. Comput. Chem. 23 (2002) 887-894.

[39] F.H. Branin Jr., IBM, J. Res. Dev. 16 (1972) 504-522.

[40] W. Quapp, D. Heidrich, J. Mol. Struct. THEOCHEM 585 (2002) 105-117.

[41] V. Bakken, D. Danovich, S. Shaik, H.B. Schlegel, J. Am. Chem. Soc. 123 (2001) 130-134.

[42] L. Windhorn, J.S. Yeston, T. Witte, W. Fuß, M. Motzkus, D. Proch, K.-L. Kompa, J. Chem. Phys. 119 (2003) 641-645. A. Papakondylis, A. Mavridis, J. Phys. Chem. A 103 (1999) 1255-1259.

[43] A.D. Issacson, in: D. Heidrich (Ed.), The Reaction Path in Chemistry: Current Approaches and Perspectives, Kluwer, Dordrecht, 1995, pp. 191-228. D.G. Truhlar, in: D. Heidrich (Ed.), The Reaction Path in Chemistry: Current Approaches and Perspectives, Kluwer, Dordrecht, 1995, pp. 191-228. T. Komatsuzaki, M. Nagaoka, Chem. Phys. Lett., 265, 1997, pp. 91-98.

[44] K. Nobusada, O.I. Tolstikhin, H. Nakamura, J. Chem. Phys. 108 (1998) 8922-8930.

[45] W. Quapp, J. Theor. Comput. Chem. 2 (2003) 385-418.

[46] K. Ruedenberg, J.-Q. Sun, J. Chem. Phys. 100 (1994) 5836-5848.

[47] D.E. Manolopoulos, K. Stark, H.-J. Werner, D.N. Arnold, S.E. Badfort, D.M. Neumark, Science 262 (1993) 1852-1855. cf. also M.H. Alexander, D.E. Manolopoulos, H.-J. Werner, J. Chem. Phys. 113 (2000) 11084-11100. and refs therein.

[48] W.S. Bian, H.-J. Werner, J. Chem. Phys. 112 (2000) 220-229. F.J. Aoiz, L. Bañares, J.F. Castillo, M. Menéndez, D. Skouteris, H.-J. Werner, J. Chem. Phys. 115 (2001) 2074-2081. Fig. 8(a).

[49] T.H. Choi, S.T. Park, M.S. Kim, J. Chem. Phys. 114 (2001) 6051-6057. 
[50] B. Lasorne, G. Dive, D. Lauvergnat, M. Desouter-Lecomte, J. Chem. Phys. 118 (2003) 5831-5840.

[51] Y.G. Smeyers, M. Villa, M.L. Senet, J. Mol. Spectrosc. 177 (1996) 66-73.

[52] J. Gonzàlez, X. Gimènez, J.M. Bofill, J. Chem. Phys. 116 (2002) 8713-8722.
[53] J. Miller, J. Furst, in: M. Nielsen (Ed.), Scale-Space 99, Lecture Notes in Computer Science, Springer, Heidelberg, 1999, pp. 93-104. J. Miller, PhD Thesis, University of North Carolina, 1998.

[54] A. Callegari, R. Pearman, S. Choi, P. Engels, H. Srivastavan, M. Gruebele, K.K. Lehmann, G. Scoles, Mol. Phys. 101 (2003) 551-568. D.J. Nesbitt, R.W. Field, J. Phys. Chem. 100 (1996) 12735-12756. 